\title{
Cortical oscillations as seizure markers in photosensitive epilepsy
}

\author{
Authors: Sanchita Bhatia, ${ }^{A}$ Megan Godfrey ${ }^{B}$ and Khalid Hamandi ${ }^{C}$
}

\section{Introduction}

Photosensitive epilepsy (PSE) is the most common reflex epilepsy, where seizures are triggered by a visual stimulus denoted by a 'photoparoxysmal response' (PPR) on an electroencephalogram (EEG). ${ }^{1}$ PSE provides a reproducible model to investigate the changes in neuronal oscillatory networks leading to interictal to ictal transition, ${ }^{2}$ and understand the pathophysiology of epilepsy overall. ${ }^{3,4}$ The aim of this study is to investigate the interictal to ictal transition in PSE using intermittent photic stimulation (IPS) by analysing cortical oscillations on scalp EEG.

\section{Methods}

Clinical EEG data with IPS, a common clinical procedure used for investigation of epilepsy, were collected for patients with genetic generalised epilepsies (GGE), and two groups were established: one with photosensitivity and another without. These data were signal processed in Matlab, epochs of IPS were extracted, PPRs were identified, and segments of EEG signal preceding the PPR were extracted from the photosensitive group. Corresponding segments from the non-photosensitive group were extracted. The various frequency components of the signal underwent a Herbert transform to produce time-frequency spectra, and average power at the stimulation frequency band and its harmonics was calculated for the frontal and occipital EEG channels. Then, the phase clustering index (PCI) was calculated to compare the phase synchrony of neurons in the photosensitive group and nonphotosensitive group.

\section{Results and discussion}

The following patient groups were included:

> GGE with photosensitivity, 28 patients (mean \pm SD age $18 \pm 12$ years)

> juvenile myoclonic epilepsy (a type of GGE), 26 patients (mean \pm SD age $22 \pm 8$ years). The average power at the stimulation frequency was increased for both the occipital and frontal channels in the photosensitive group vs non-

Authors: ${ }^{A}$ Cardiff University; ${ }^{B}$ Cardiff University Brain Research Imaging Centre (CUBRIC); ${ }^{C}$ Epilepsy Unit, University Hospital of Wales photosensitive, indicating that there is a greater degree of entrainment or locking of neuronal oscillation to the visual stimulus, albeit not statistically significant (frontal: $p=0.1187$, occipital: $p=0.0595$, unpaired t-test adjusted for multiple comparisons). There was also significantly increased phase clustering for frontal and occipital channels (channel F4: $p=0.0195, F 7: p=0.0174,02: p=0.0174$, unpaired $t$-test adjusted for multiple comparisons).

\section{Conclusions}

The enhanced phase synchronisation and degree of entrainment of neuronal oscillations can be used as potential biomarkers of epileptic transition in PSE. These results help understand mechanisms of underlying perceptual processes involved in the pathophysiological changes that occur in brain networks in epilepsy, to improve diagnosis and develop effective treatment in the future.

\section{References}

1 Kalitzin S, Parra J, Velis DN, Lopes Da Silva FH. Enhancement of phase clustering in the EEG/MEG gamma frequency band anticipates transitions to paroxysmal epileptiform activity in epileptic patients with known visual sensitivity. IEEE Trans Biomed Eng 2002:49:1279-86.

2 Lopes Da Silva FH, Harding GFA. Transition to seizure in photosensitive epilepsy. Epilepsy Res 2011;97:278-82.

3 Parra J, Kalitzin SN, Iriarte ] et al. Gamma-band phase clustering and photosensitivity: is there an underlying mechanism common to photosensitive epilepsy and visual perception? Brain 2003:126:1164-72.

4 Perry G, Brindley LM, Muthukumaraswamy SD, Singh KD, Hamandi K. Evidence for increased visual gamma responses in photosensitive epilepsy. Epilepsy Res 2014;108:1076-86. 